Document downloaded from:

http://hdl.handle.net/10251/166524

This paper must be cited as:

Wang, S.; Cabrero-Antonino, M.; Navalón Oltra, S.; Cao, C.; Tissot, A.; Dovgaliuk, I.; Marrot, J.... (2020). A Robust Titanium Isophthalate Metal-Organic Framework for Visible-Light Photocatalytic CO2 Methanation. Chem. 6(12):3409-3427. https://doi.org/10.1016/j.chempr.2020.10.017

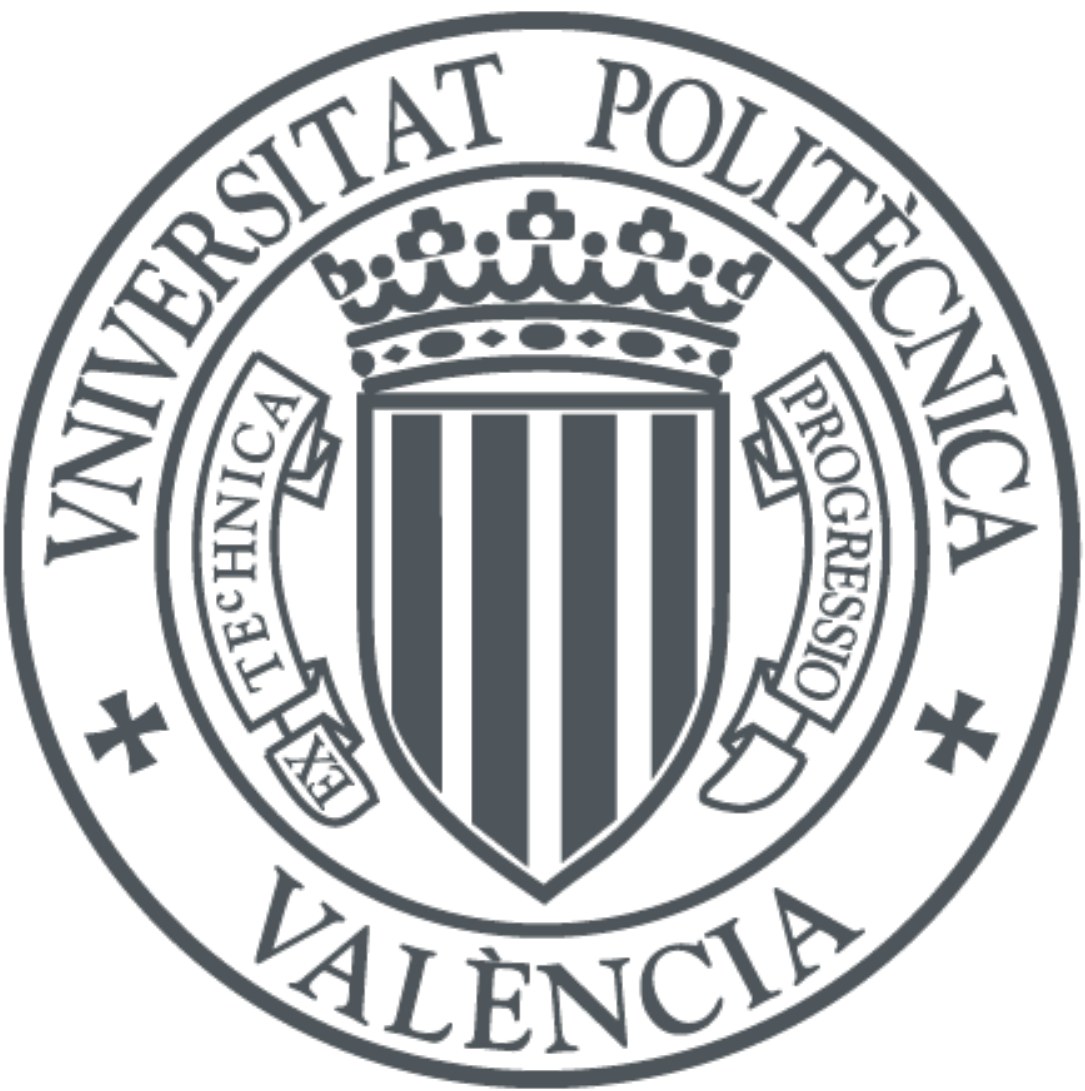

The final publication is available at

https://doi.org/10.1016/j.chempr.2020.10.017

Copyright Elsevier (Cell Press)

Additional Information 


\section{A Robust Titanium Isophthalate Metal-Organic Framework for Visible Light Photocatalytic $\mathrm{CO}_{2}$ Methanation}

Sujing Wang ${ }^{1,2,8 *}$, Maria Cabrero-Antonino ${ }^{3}$, Sergio Navalón ${ }^{3}$, Chen-chen Cao ${ }^{1}$, Antoine Tissot ${ }^{1}$, Iurii Dovgaliuk ${ }^{1}$, Jérôme Marrot $^{4}$, Charlotte Martineau-Corcos ${ }^{4,5}$, Liang $\mathrm{Yu}^{6}$, Hao Wang ${ }^{6}$, William Shepard ${ }^{7}$, Hermenegildo García ${ }^{3 *}$, Christian Serre $^{1 *}$ ${ }^{1}$ Institut des Matériaux Poreux de Paris, UMR 8004 CNRS, Ecole Normale Supérieure, Ecole Supérieure de Physique et de Chimie Industrielles de Paris, Université PSL, 75005 Paris, France

${ }^{2}$ CAS Key Laboratory of Microscale Magnetic Resonance, Hefei National Laboratory for Physical Sciences at the Microscale, University of Science and Technology of China, 230026, Hefei, China

${ }^{3}$ Departamento de Química, Universitat Politècnica de València, 46022, Valencia, Spain

${ }^{4}$ Institut Lavoisier de Versailles, UMR 8180 CNRS, Université de Versailles Saint-Quentin-en-Yvelines, Université Paris-Saclay, 78035 Versailles, France

${ }^{5}$ CEMHTI, UPR 3079 CNRS, 45071 Orléans CEDEX 2, France

${ }^{6}$ Hoffmann Institute of Advanced Materials, Shenzhen Polotechnic, 518055, Shenzhen, China.

${ }^{7}$ Synchrotron SOLEIL, L’Orme des Merisiers, Saint-Aubin, BP 48, 91192, Gif-Sur-Yvette, France

${ }^{8}$ Lead contact

*Correspondence:

sjwang4@ustc.edu.cn (S.W.)

hgarcia@qim.upv.es (H.G.)

christian.serre@ens.fr (C.S.) 


\section{SUMMARY}

Isophthalic acid (IPA) has been considered to build metal-organic frameworks (MOFs), owing to its facile availability, unique connection angle/mode and a wide scope of functional groups attached. Constructing titanium-IPA frameworks that possess photoresponse properties is an alluring characteristic, in relation to the challenge of synthesizing new Ti-MOFs. Here, we report the first Ti-IPA MOF (MIP-208), that efficiently combines the use of preformed $\mathrm{Ti}_{8}$ oxoclusters and in situ acetylation of the 5- $\mathrm{NH}_{2}$-IPA linker. The mixed solid-solution linkers strategy was successfully applied resulting in a series of multivariate MIP-208 structures with tunable chemical environments and sizable porosity. MIP-208 shows the best result among the pure MOF catalysts for the photocatalytic methanation of carbon dioxide $\left(\mathrm{CO}_{2}\right)$. To improve the photocataytic performance, ruthenium oxide nanoparticles were photodeposited on MIP-208 forming a highly active and selective composite catalyst, MIP-208@ $\mathrm{RuO}_{\mathrm{x}}$, which features a notable visible light response, an excellent stability and recycling ability. 


\section{INTRODUCTION}

Metal-organic frameworks (MOFs) or porous coordination polymers (PCPs) have been recognized as one of the most intensively investigated heterogeneous catalysts for liquid phase reactions, due to their porous tunable structure, high surface area, and acidic/basic sites or metal nodes with versatile and spatially controlled distributions. ${ }^{1-3}$ Organic ligands play an essential role in the formation of the MOF structure and the introduction of its corresponding property. ${ }^{4 ;}$ As organic synthesis has evolved significantly over the last few decades, an almost unlimited number of organic compounds have been applied to MOF fabrication via the linker-engineering strategy, resulting in a vast combination of structures and functions. ${ }^{6-9}$ However, the time and effort requiring organic synthesis for linker preparation undoubtedly limits the reproduction and application of costly MOF candidates. ${ }^{10 ;} 11$ In this regard, commercially available organic linkers from industry are of great interest due to their ready availability on a large scale and low cost. ${ }^{12 ; 13}$

Benzene-dicarboxylic acid isomers are representative compounds that fulfill the aforementioned demand currently produced on a billion-kilogram scale per annum. The three isomers, namely ortho-phthalic acid (phthalic acid), meta-phthalic acid (isophthalic acid, IPA) and para-phthalic acid (terephthalic acid), are all important feedstocks for the preparation of a large number of industrial products. ${ }^{14 ;}{ }^{15}$ In their use of constructing MOFs, phthalic acid alone tends to form materials of low dimensionality, mainly due to the deficient separation and steric hindrance between the adjacent carboxylate groups. ${ }^{16 ; 17}$ On the contrary, terephthalic acid is one of the most popular carboxylate linkers since the very beginning of MOF science. ${ }^{18}$ Its ideal configuration with a suitable molecular length, perfect separation of the coordination sites and varied connection modes, make this linker adaptable to MOF structures with any dimension, and built with almost every sort of metal element. ${ }^{19-21}$

In the case of isophthalic acid, the unique angle $\left(120^{\circ}\right)$ and the medium distance between the two carboxylate groups enable a large degree of manipulation in the 
synthesis of MOFs compared with the other two isomers. Numerous metal-IPA frameworks have been identified with nodes ranging from the alkaline earth metals, ${ }^{22}$ to divalent and trivalent transition metals, ${ }^{23-26}$ as well as rare earth ${ }^{27-29}$ or main group elements, ${ }^{30-32}$ and even mixed metal systems, ${ }^{33-35}$ in which the meta-side of the coordinated carboxylate groups on the benzene ring frequently faces the structure void. IPA offers thus an obvious and facile opportunity of tuning the chemical environment of the MOF pore via the introduction of functional groups on the meta-side. Correspondingly, distinct organic functions on the 5- and 4-position of the benzene ring have been reported, showing great interest and potential in fine-tuning the structureproperty correlation. ${ }^{36-39}$

It is noteworthy that isophthalic acid is suitable for MOF assembly with every transition metal subgroup except for the Group 4 elements in the periodic table, and to the best of our knowledge, there has been no report of IPA based-MOF examples built with titanium (Ti), zirconium (Zr), hafnium (Hf) or rutherfordium (Rf). On the other hand, $\mathrm{Ti}$ and $\mathrm{Zr}$, the most common Group 4 metals, have attracted much attention from the MOF research community during the past years, due to their high natural abundance, low toxicity, strong interactions with oxygen sites and thus significantly enhanced stabilities once in the MOF structures..$^{40 ; 41}$

More recently, the potential of MOFs has expanded into other areas of catalysis, particularly in photocatalysis. ${ }^{42}$ The strong interaction between the positively charged metal nodes and negatively charged antenna linkers in rigid configurations makes MOFs suitable for the promotion of photoinduced charge separation. ${ }^{42}$ Hence, an electron transfer from the organic linker in its excited state to the metal node upon light absorption appears to be a general photochemical step, especially in the case of a favorable overlap between the lowest occupied crystal orbit (LOCO) with the atomic orbitals of the metal cations. ${ }^{43}$ For this reason, MOFs are among the most promising category of porous photocatalysts, particularly for the production of solar fuels.

Taking into account the prevalent role of MOFs and derivative materials in 
photocatalysis, Ti-MOFs have attracted considerable attention due to the known activities of $\mathrm{TiO}_{2}$ and Ti-oxo clusters to undergo photoinduced charge separation. ${ }^{44}$ In general, Ti-MOFs are not only promising candidates as photo-responsive materials, ${ }^{45}$ but also show great potential in gas separation, ${ }^{46 ; 47}$ proton conduction ${ }^{48}$ and clinical applications. ${ }^{49}$ Nevertheless, direct synthesis of Ti-MOFs still remains an important challenge ${ }^{50}$ despite the gradually increasing numbers of reported Ti-MOF structures over the past decade, which have been attained mostly through serendipity. It is expected that an infinite number of interconnected Ti-O units would facilitate photoinduced charge separation and charge mobility, in comparison with MOFs based on discrete inorganic units with a low number of Ti-O bonds. One and two dimensional (1D and 2D) arrays of Ti-O units should thus in principle improve the semiconducting properties of the Ti-MOFs. ${ }^{51}$ In this context, the photoactive Ti-MOFs that have been prepared so far are those with 1D chains or nanowires of Ti-O building units, whose photo-responsive behavior have been proven to be closer to those compounds constructed of discrete Ti-O clusters than that of bulk $\mathrm{TiO}_{2} \cdot{ }^{52}$ As shown in Figure 1, there are only three kinds of 1D Ti-O inorganic building units reported to date, namely the TiO chains in MIL-91, ${ }^{53}$ the $\mathrm{Ti}_{6} \mathrm{O}_{9}$ nanowires in MIP-177-HT, ${ }^{52} \mathrm{ZSTU}-1,2$ and $3,{ }^{54}$ and the TiO chain in DGIST-1. 55 Therefore, as these MOFs are all constructed from sophisticated ligands, it would be of great interest to synthesize IPA type linker-based Ti-MOFs with infinite Ti-O building units. It would not only expand the scope of TiMOFs structural and chemical diversity, but also may lead to improved photocatalytic performances.

Herein, we report an ultramicroporous Ti-MOF, denoted as MIP-208 (MIP stands for the Materials of the Institute of porous materials from Paris), the first MOF composed of Group 4 metal and IPA type linker. In the crystal structure of MIP-208, helical chains of cis-connected corner-sharing $\mathrm{TiO}_{6}$ polyhedra are held in place by the in situ generated 5-acetamidoisophthalate (5-Aa-IPA) linker molecules, giving rise to a 3D framework with 1D channel-like accessible voids running along the $c$-axis. Benefiting from their shared structural configuration and coordination adaptability, mixed linkers 
of 5-Aa-IPA and other IPA derivatives with diverse functional groups on the metaposition of the carboxylate groups could be further introduced in the framework following a solid-solution strategy, resulting in a series of multivariate MIP-208 structures. Furthermore, the dual presence of the amide group and the $1 \mathrm{D} \mathrm{TiO}$ chain unit in the porous structure of MIP-208, associated with an excellent thermal and hydrothermal stability, has allowed the development of a photocatalyst for the methanation of carbon dioxide $\left(\mathrm{CO}_{2}\right)$ based on $\mathrm{RuO}_{\mathrm{x}}$ nanoparticles (NPs) under visible light, reaching a productivity of $0.8 \mathrm{mmol} \mathrm{g}_{\text {catalyst }}{ }^{-1}$ at $200{ }^{\circ} \mathrm{C}$ in $22 \mathrm{~h}$.

\section{RESULTS AND DISCUSSION}

Twenty-four different Ti-MOFs prepared from direct-synthesis have been reported to date. The Ti sources used to produce these MOFs can be classified into two kinds: simple Ti complexes and polynuclear TiO clusters. Compared with simple Ti complexes, such as $\mathrm{TiCl}_{3}, \mathrm{TiCl}_{4}$, and $\mathrm{Ti}(\mathrm{iPrO})_{4}$, Ti-oxoclusters are known to slow down the reaction rate and avoid the undesirable hydrolytic formation of $\mathrm{TiO}_{2}$, owing to the gradual rearrangements of Ti-O connections of the oxocluster precursor for most cases. Thus, Ti-oxoclusters usually lead to highly crystalline Ti-MOF materials composed of large single crystals. ${ }^{55-57}$ In this regard, a $\mathrm{Ti}_{8} \mathrm{O}_{8}$ cluster with formate and acetate terminals ( $\left.\mathrm{Ti}_{8} \mathrm{AF}\right)$ was selected here as a reactant, due to its facile preparation, easy handling, suitable stability and reactivity. ${ }^{58}$ Regarding solvent for the reaction, it is wellrecognized that the conventional ones, such as dimethylformamide (DMF) and diethylformamide (DEF), should be replaced by greener solvents to decrease the toxicity to humans and the environment. Based on our previous success of preparing Group 4 metal MOFs, formic acid, acetic acid and acetic anhydride, which have been shown to efficiently slow down the reaction leading to highly crystalline products, were selected as solvents. 5- $\mathrm{NH}_{2}$-IPA was selected as the linker due to the documented benefit of the amino group presence in increasing the $\mathrm{CO}_{2}$ adsorption and enhancing the visible light photoresponse..$^{59 ; 60}$

Initial reaction attempts indicated that the aforementioned three acidic solvents can 
interfere with decomposition of the Tis $\mathrm{AF}$ precursor, while offering a good solubility for the 5- $\mathrm{NH}_{2}$-IPA linker. However, all attempts did not generate any crystalline solid. Various additives known to either facilitate the linker exchange or promote the rearrangement of Ti-O connections were tested, such as water, methanol, ethanol, acetone, and DMF. A highly crystalline product of a dark yellow or light brown color was formed only when methanol was used in the mixture of acetic acid and acetic anhydride. Acetic acid was found to be not necessary for the product formation, but to accelerate the reaction rate. An in situ amidation took place between the amino group of 5- $\mathrm{NH}_{2}$-IPA and acetic anhydride, generating the 5-acetamide-IPA (5-Aa-IPA) as the final framework building linker (Figure S1). Solid state NMR data collected on the assynthesized MIP-208 sample demonstrated the complete conversion of 5- $\mathrm{NH}_{2}$-IPA to 5-Aa-IPA, which serves as the linker to build the MOF structure (Figures S2-S4).

It is likely that the in situ acetylation step is critical to yield the crystalline material, which possibly slows down the reaction rate significantly. A control experiment using the preformed 5-Aa-IPA as linker under the same reaction conditions did not lead to MIP-208, but to an amorphous solid. Therefore, this solvothermal synthesis is likely to benefit synergistically from the rearrangement of the $\mathrm{Ti}_{8} \mathrm{AF}$ cluster and in situ linker formation, which is a rare case for MOF preparation reported so far. However, it could provide a valuable and even rational strategy for the synthesis of new Ti-MOFs through a control of the reaction rate from both inorganic and organic precursor conversions.

MIP-208 was obtained as micro-sized single crystals, ruling out its structure determination from laboratory diffraction. Thus single-crystal X-ray diffraction data of MIP-208 were collected with microfocused X-rays on the synchrotron beamline PPOXIMA 2A (Synchrotron SOLEIL, France) ${ }^{61}$ to determine its crystal structure. The coordination environment of the $\mathrm{Ti}^{4+}$ ion and the overall framework connection could be solved precisely, even though the position of the acetamide group could not be accurately located due to its disorder. Assisted by the solid-state NMR characterization, an empirical formula of $\operatorname{Ti}\left(\mu_{2}-\mathrm{O}\right)(5-\mathrm{Aa}-\mathrm{IPA})$ could be dertermined for MIP-208. Rietveld refinement carried out on the high resolution powder X-ray diffraction data 
(PXRD) of MIP-208 confirms the aforementioned structural model (Figure S5). It crystallizes in tetragonal $I 4_{1} /$ amd space group (No. 141) with the unit-cell constants $a$ $=b=21.8190 \AA$ and $c=10.9380 \AA$. As shown in Figure $2 \mathrm{~A}$, each $\mathrm{Ti}^{4+}$ ion is in an octahedral coordination geometry with four carboxylate oxygen atoms and two $\mu_{2}-\mathrm{O}$ oxo groups. The adjacent $\mathrm{TiO}_{6}$ polyhedra adopt a cis-connected corner-sharing mode to connect each other, giving rise to a $1 \mathrm{D}$ infinite chain of secondary building units (SBUs) running along the $c$-axis. Unlike the linear 1D Ti-O SBUs in MIL-91, ${ }^{53}$ DGIST- $1^{55}$ and MIP-177-HT, ${ }^{52}$ the helical TiO chain in MIP-208 is the first example of this type of SBU observed in Ti-MOFs, to our knowledge (Figure 1). An $\mathrm{Al}(\mathrm{OH})$ chain with a similar configuration was discovered previously in the CAU-10 MOF series (CAU stands for Christian-Albrechts-University), ${ }^{62}$ with $\mu_{2}-\mathrm{OH}$ group as the shared corner.

Notably, the replacement of the bridging $\mathrm{OH}$ in trivalent metal-based MOFs by an oxo group in similar MOFs built with tetravalent metals could lead to a dramatic difference in terms of structural flexibility and related properties, as documented in MIL-47 and MIL-53 compounds (MIL stands for Materials from Institute Lavoisier). ${ }^{63 ;} 64$ In particular, the presence of $\mathrm{OH}$ or oxo groups in Ti-O materials has been demonstrated to be critical to their performance in photocatalysis, ${ }^{65-67}$ which highlights the importance of TiO chains in MIP-208 structure over its analogues with the lower valence metal ions.

The neighboring $\mathrm{TiO}$ chains are interconnected via pairs of 5-Aa-IPA linkers in an 'up and down' mode to avoid as much as possible any steric hindrance (Figure 2B), resulting in a 3D framework with double-walled $1 \mathrm{D}$ channels running along the $c$-axis (Figure 2C). One can expect its micropores to be accessible for guest molecules as reported before for CAU-10 compounds constructed from IPA bearing the bulkiest functional groups, ${ }^{62 ; 68}$ due to the large size and thus considerable steric hindrance of the acetamide groups facing the pores. As reported before for CAU-10-OMe and CAU10-Br, MIP-208 did not show any accessibility for nitrogen at $77 \mathrm{~K}$, but a noticeable porosity for $\mathrm{CO}_{2}$ (Figure $\mathrm{S} 4$ ).

A large number of functionalized IPAs are commercially available chemicals offered at 
low prices. The high compatibility of IPA derivatives regarding their behavior in coordination chemistry has been documented, ${ }^{37}$ thus offering the possibility to tune the chemical environment of the pore in the MIP-208 structure via a solid-solution mixed linker strategy. The solid-solution synthesis strategy is a powerful alternative way to introduce multiple functional groups together in MOF structures, especially when the direct synthesis of the isostructural MOF with the single linker bearing specific functional group as a reactant is challenging. ${ }^{69-71}$ This strategy suits perfectly the case of MIP-208, since all attempts to directly synthesize the corresponding MIP-208 analogues using other functionalized IPAs instead of 5- $\mathrm{NH}_{2}$-IPA have failed up to now, resulting in either amorphous solids or crystalline phases of different structures.

Therefore, mixed linkers were considered via one-pot reactions to prepare a series of multivariate MIP-208s (MTV-MIP-208, Figure 3A). Several representative IPA-type dicarboxylic acids, including IPA, 5- $\mathrm{NO}_{2}$-IPA, 5-Me-IPA, 5-Br-IPA, 5-tBu-IPA, 3,5pyrazole-dicarboxylic acid (PDA) and camphoric acid, were selected as the substitution linkers, aiming to replace the 5-Aa-IPA spacer in the MIP-208 framework. All the tested linkers except for 5-NO $\mathrm{NO}_{2}$-IPA and camphoric acid could reach certain substitution ratios, ranging from $11 \mathrm{~mol} \%$ to $50 \mathrm{~mol} \%$ (Figure S8), as deduced from the NMR data. However, the reason for the unsuccessful inclusion of 5-NO $-\mathrm{NI}_{2} \mathrm{IP}$ and camphoric acid is not yet clear and still under investigation.

As shown in Figure 3B, the inclusion of the second linker did not generate noticeable changes on the overall structural long-range order, as evidenced by their highly similar PXRD patterns. However, the porosities of the resulting materials were altered, as deduced from the $\mathrm{CO}_{2}$ adsorption isotherms collected at $273 \mathrm{~K}$ (Figure 3C). For instance, the sample with $50 \mathrm{~mol} \%$ IPA substitution ratio led to a significant enhancement of $\mathrm{CO}_{2}$ uptake (50\% at both 0.15 and 1 bar) compared with the pure MIP208, likely because of the smaller $\mathrm{H}$ atoms providing less steric hindrance over the larger acetamide group. A similar observation was noticed for the 5-Me-IPA mixed sample (33 mol\%). In the case of the 5-Br-IPA derivative (38 mol\%), a slight decrease of uptake in comparison with the parent compound has been observed, possibly 
attributable to the much heavier atomic weight of the $\mathrm{Br}$ atom, in spite of its lower steric hindrance. When the slightly bulkier $t \mathrm{Bu}$ group, but of similar molecular weight, was introduced to replace the acetamide group, the sample shows a limited drop in pore volume mainly due to the small linker substitution ratio $(17 \mathrm{~mol} \%)$. It is worth noting that even PDA, which has a five-membered heterocyclic aromatic moiety, could be included in the MIP-208 framework, though the substitution ratio is low (11 mol\%), probably due to the small change in the angle of the two carboxylate groups and the presence of basic $\mathrm{N}$-site on the heterocyclic ring. Therefore, solid-solution synthesis strategy here is an efficient way to expand and modify the chemical variety of the MIP208 structural cavity, leading to a series of MTV-MIP-208 materials.

The stability of any MOF is a practical concern in its applications. For instance, photocatalytic methanation requires a combination of thermal, hydrolytic and UV irradiation stability of the MOF catalyst, since the catalysis is normally carried out at temperatures close to $200{ }^{\circ} \mathrm{C}$, under UV irradiation and in the presence of stoichiometric water generated as the side product. Therefore, the stability of MIP-208 was tested before applying it to the photocatalytic methanation. The results from both temperature-dependent PXRD measurements and thermogravimetric analysis (TGA) support the high stability of MIP-208 (Figure S9). Its ordered crystal structure is stable up to $325^{\circ} \mathrm{C}$ in air, which is comparable to MIP-177-HT, and clearly surpasses MIL91 and DGIST-1 (unstable above $200^{\circ} \mathrm{C}$ ). Furthermore, the activated MIP-208 sample hardly adsorbs water (less than $5 \mathrm{wt} \%$ ) when exposed to air for a long time, considerably much less than the Al-CAU-10 series do. This enhanced hydrophobicity of MIP-208 is thus associated to the bridging oxo group effect, in comparison with the $\mathrm{OH}$ in CAU10 , as well as the limited hydrophilic character of acetamide.

Soaking MIP-208 in liquid water at room temperature for three days and heating it in boiling water for $8 \mathrm{~h}$, separately, tested its hydrolytic stability. The PXRD patterns of the tested samples are identical to those of the as-synthesized ones (Figure 4A), supporting the robustness of the crystalline long-range order. The $\mathrm{CO}_{2}$ adsorption data (Figure 4B) confirm the high hydrolytic stability of MIP-208, with minor differences 
between the sample before and after water treatments. In addition, the hydrolytic stability of MIP-208 in aqueous solution with various $\mathrm{pH}$ values were also investigated, which supports the excellent resistance of MIP-208 towards those chemical treatments (Figure S10). It is worth noting that MIP-208 is among the most hydrothermally stable Ti-MOFs (comparable to that of the Ti-bisphosphonate MIL-91) and outperforms all Ti-carboxylate MOFs reported so far.

As MIP-208 possesses 1D TiO chain building block, a good photoresponsive activity is expected. Photocatalytic conversion of $\mathrm{CO}_{2}$ to methane was thus selected as the model reaction, since this reaction could generate value-added product from $\mathrm{CO}_{2}$ emissions. Promising progress has been achieved lately in using MOF-based heterogeneous catalysts for $\mathrm{CO}_{2}$ conversion to methane. ${ }^{72}$ For instance, MOFs supported nickel and platinum nanoparticles (NPs) could promote the thermal methanation under harsh conditions, such as high pressures and temperatures. Photocatalytic methanation by MOF-based catalysts has, however, been hampered for a long time by poor selectivity until the recent success reported of a HKUST-1@ $\mathrm{TiO}_{2}$ core-shell composite applied under UV irradiation, albeit its rather low efficiency. ${ }^{73}$ Lately, the first example of a pure photoactive MOF displaying catalytic methanation has been reported, which involves $\mathrm{ZnO}$ inorganic bricks in a nitrogen-rich framework exhibiting a $\mathrm{CH}_{4}$ generation productivity of $30 \mu \mathrm{mol} \cdot \mathrm{g}_{\text {catalyst }}{ }^{-1}$ after $24 \mathrm{~h}$ at $215^{\circ} \mathrm{C}$ under UV light irradiation. A notable enhancement of activity was attained via supporting $\mathrm{Cu}_{2} \mathrm{O}$ NPs on the $\mathrm{Zn}-\mathrm{MOF}$ to form a composite catalyst, which led to a $\mathrm{CH}_{4}$ production of $45 \mu \mathrm{mol} \cdot \mathrm{g}_{\text {catalyst }}{ }^{-1}$ under the same conditions. ${ }^{74}$ These breakthroughs are undoubtedly encouraging, although the photocatalytic activity of MOF materials remains very low. Therefore, developing efficient and selective MOF-based photocatalysts for methanation is of great interest, but still remains a challenge.

Pure MIP-208 samples were first tested for photocatalytic methanation. Preliminary control experiments under similar conditions as those used in the photocatalytic reaction, but in the dark, showed that MIP-208 did not promote thermally $\mathrm{CO}_{2}$ reduction to $\mathrm{CH}_{4}$. In contrast, a $\mathrm{CH}_{4}$ production of about $40 \mu \mathrm{mol} \mathrm{g}_{\text {catalyst }}{ }^{-1}$ could be achieved using 
MIP-208 as the catalyst in the mixture of $\mathrm{CO}_{2}$ (0.25 bar) and hydrogen (1.05 bar) under UV-Vis irradiation (Xenon lamp, $1350 \mathrm{~W} \times \mathrm{m}^{-2}$ ) at $200{ }^{\circ} \mathrm{C}$ after 22 hours (Figure S11). This result highlights the positive influence of the 1D TiO chain SBU in MIP-208 regarding its photo-response, since the benchmark compound $\mathrm{NH}_{2}-\mathrm{MIL}-125$, which is built with discrete $\mathrm{Ti}_{8}$ clusters, exhibits negligible photocatalytic activity under similar reaction conditions. ${ }^{74}$ Furthermore, in comparison with the state-of-the-art MOF photocatalysts for methanation, ${ }^{74}$ an enhancement of $33 \%$ in efficiency was noticed for MIP-208, suggesting that MIP-208, to our knowledge, could be the most efficient pure MOF photocatalyst for methanation to date.

In order to further improve the $\mathrm{CH}_{4}$ formation rate, incorporation of metal NPs onto MIP-208 was considered to form a composite catalyst, since it is well-documented that photocatalytic methanation generally requires the presence of a metal or metal oxide with hydrogenation activity, such as nickel, copper, rhodium, palladium, or ruthenium. ${ }^{75}$ We initially selected $\mathrm{RuO}_{\mathrm{x}} \mathrm{NPs}$ as the composite component, owing to the fact that $\mathrm{Ru}$ species generally show the highest activity in the methantion of $\mathrm{CO}_{2}$ under milder conditions, despite their limited selectivity compared with other active elements. ${ }^{76 ; 77}$ In addition, the oxide form, instead of elemental metal NPs, features an elevated stability to facilitate easy handling during catalysis process. The MIP$208 @ \mathrm{RuO}_{\mathrm{x}}$ composite was obtained following the post-synthetic photo-deposition of $\mathrm{RuO}_{\mathrm{x}}$ NPs on MIP-208 by the photocatalytic reduction of water-soluble $\mathrm{KRuO}_{4}$ using methanol as the sacrificial electron donor (Figure S12). The success of $\mathrm{RuO}_{\mathrm{x}}$ photodeposition by photocatalytic perruthenate reduction further demonstrates the photoactivity of MIP-208, as it has been proven that an evidence supporting the occurrence of photoinduced charge separation upon irradiation of a solid is the observation of photodeposition onto the material of metal NPs from aqueous solutions. $^{78}$

After deposition, determination of the $\mathrm{Ru}$ content and average particle size in the composite was carried out. Energy-dispersive X-ray spectroscopy (EDX) analysis of MIP-208@ $\mathrm{RuO}_{\mathrm{x}}$ showed a Ru content of $0.76 \mathrm{wt} \%$. Field emission scanning electron 
microscopy (FESEM) images of MIP-208@ $\mathrm{RuO}_{\mathrm{x}}$ displayed a similar crystallite morphology as that of the pristine MOF, without obvious free $\mathrm{RuO}_{\mathrm{x}}$ particles (Figure S13). Dark-field scanning transmission electron microscopy (DF-STEM) measurements revealed the presence of small $\mathrm{RuO}_{\mathrm{x}}$ NPs $(1.4 \pm 0.14 \mathrm{~nm})$. The homogeneous distribution of $\mathrm{RuO}_{\mathrm{x}}$ NPs all over MIP-208 was further confirmed by DF-STEM coupled to an EDX detector (Figure S14).

Preservation of the crystalline structure of MIP-208 after $\mathrm{RuO}_{\mathrm{x}}$ photodeposition was confirmed by PXRD data (Figure S15). The lack of additional peaks in the composite pattern compared with that of the pure MIP-208 could be attributed to the low $\mathrm{Ru}$ loading and the evenly distributed small particle size, as determined by TEM images.

The MIP-208@ $\mathrm{RuO}_{\mathrm{x}}$ solid was also characterized by X-ray photoelectron spectroscopy (XPS, Figure S16). The C1s spectrum shows the presence of aromatic carbons (284.4 $\mathrm{eV}$ ). In addition, a band centred at $285.9 \mathrm{eV}$ attributable to the $\mathrm{C}-\mathrm{N}$ bond of the amide groups is observed, together with another centred at $289 \mathrm{eV}$ that can be assigned to the carbonyl of both the amide and the carboxylate groups. The amide group is clearly observed in the N1s band centred at about $399 \mathrm{eV}$ as well. The broad O1s band is mainly due to the presence of oxygen atoms in the carboxylate and amide groups, together with the $\mathrm{Ti}-\mathrm{O}$ and $\mathrm{Ru}-\mathrm{O}$ bonds. $\mathrm{Ti} 2 \mathrm{p}$ spectrum can be assigned to the presence of $\mathrm{Ti}^{4+}$ ions. Ruthenium is recognized by the bands appearing at about 280.7 and $282.4 \mathrm{eV}$ that can be assigned to the $\mathrm{Ru} 3 \mathrm{~d}_{5 / 2}$ of $\mathrm{RuO}_{2}$ and hydrated $\mathrm{RuO}_{2}$, respectively. ${ }^{79} 80$ The expected $\mathrm{Ru} 3 \mathrm{~d}_{3 / 2}$ band appearing at higher binding energies ( 4.1 eV) (Figure S16) is, however, difficult to observe, since it overlaps with the $\mathrm{C}-\mathrm{N}$ contribution of the acetamido groups ${ }^{78 ; 80}$ XPS analysis also can provide an estimation of the valence band maximum of the MIP-208@ $\mathrm{RuO}_{\mathrm{x}}$ solid by measuring the lowest energy electron band of the material referred to the work function of the instrument. As shown in Figure S17, a valence band potential of $-1.51 \mathrm{~V}$ was estimated. This value together with the $2.92 \mathrm{eV}$ bandgap measured from the Tauc plot of the diffuse reflectance UV-Vis spectrum, gives a value for the LUCO of $-2.874 \mathrm{~V}$. 
The photocatalytic performance of the MIP-208@RuOx composite in the methanation of $\mathrm{CO}_{2}$ was then evaluated under the same reaction conditions as that used for testing the pristine MOF. Control experiments using MIP-208@ $\mathrm{RuO}_{\mathrm{x}}$ in the dark under the same reaction condition showed negligible $\mathrm{CH}_{4}$ production. As expected, a selective $\mathrm{CO}_{2}$ conversion into $\mathrm{CH}_{4}\left(800 \mu \mathrm{mol} \mathrm{g}\right.$ catalyst $\left.^{-1}\right)$ as the only product was observed (Figure $5 \mathrm{~A})$. Specifically, the amounts of $\mathrm{CO}$ and $\mathrm{C}_{2} \mathrm{H}_{6}$ were under the detection limit of our micro-GC. This $\mathrm{CH}_{4}$ production value is 20 times higher compared with that obtained using the pure MIP-208 sample. Regarding product selectivity, photocatalytic $\mathrm{CO}_{2}$ hydrogenation renders generally methane or $\mathrm{CO}$ as the main products, accompanied by minor amounts of $\mathrm{C} 2$ and $\mathrm{C}^{81}$. Selectivity to methane or $\mathrm{CO}$ depends mainly on the strength of $\mathrm{CO}$ adsorption and the hydrogenating activity of the photocatalysts. In general, if CO remains strongly adsorbed on the photocatalyst, further hydrogenation takes place and the final product is methane. It is proposed that MIP-208 favors CO adsorption near the $\mathrm{RuO}_{\mathrm{x}}$ active sites and thus leads to high methane selectivity.

Similar time-conversion plots, with a slightly lower $\mathrm{CH}_{4}$ formation rate, were also measured using simulated sunlight irradiation (1 Sun) (Figure 5A). The difference between the $\mathrm{CH}_{4}$ production using a xenon lamp or simulated sunlight can be attributed to the lower power of the simulated sunlight $\left(1000 \mathrm{~W} \times \mathrm{m}^{-2}\right)$ compared to the xenon lamp $\left(1350 \mathrm{~W} \times \mathrm{m}^{-2}\right)$. Furthermore, photomethanation of $\mathrm{CO}_{2}$ using MIP-208@RuOx as catalyst was also performed upon visible light irradiation by filtering the output beam of the Xe lamp $(\lambda>455 \mathrm{~nm})$. A similar temporal $\mathrm{CH}_{4}$ formation profile was recorded, with a $\mathrm{CH}_{4}$ production at final time for visible light about $78 \%$ respect to the use of the full UV-Vis light emission provided by the Xe lamp (Figure 5A). This similarity in the temporal $\mathrm{CO}_{2}$ conversion indicates that a major percentage of the MIP-208@ $\mathrm{RuO}_{\mathrm{x}}$ photoresponse must derive from the visible region. Both experiments under simulated sunlight illumination and visible light irradiation indicate that a considerable proportion of the MIP-208- $\mathrm{RuO}_{\mathrm{x}}$ photoresponse derives from the visible region. This photoresponse indicates that $\mathrm{RuO}_{\mathrm{x}}$ is acting as light harvester in addition as active site, since MIP-208 in the absence of $\mathrm{RuO}_{\mathrm{x}}$ exhibits much less photomethanation rate and 
should not be responsive under visible light.

The recycling stability of the MIP-208@ $\mathrm{RuO}_{\mathrm{x}}$ composite as a photocatalyst was determined by performing a series of consecutive recycling runs following the temporal evolution of $\mathrm{CO}_{2}$ conversion and $\mathrm{CH}_{4}$ formation. These stability tests are important considering the conditions of photocatalytic methanation, particularly the presence of $\mathrm{H}_{2} \mathrm{O}$ and the reaction temperature of $200{ }^{\circ} \mathrm{C}$. The results presented in Figure $5 \mathrm{~B}$ show coincident plots for the temporal $\mathrm{CH}_{4}$ evolution upon seven consecutive runs, supporting the stability of the material under the conditions of the photocatalytic reaction. It is noteworthy that this highly selective and constant methanation activity promoted by $\mathrm{RuO}_{\mathrm{x}}$ has hardly been achieved before, as $\mathrm{CO}$ has been proven to be the side product in this reaction, which led to a considerable deactivation of the catalyst. ${ }^{77}$ Additionally, irradiation of the MIP-208@ $\mathrm{RuO}_{\mathrm{x}}$ composite in argon atmosphere using the xenon lamp or solar simulator at $200{ }^{\circ} \mathrm{C}$ revealed the evolution of a tiny amount of $\mathrm{CO}_{2}$, corresponding to the decomposition of a small fraction of the IPA linkers present in the material $\left(<0.05 \mu \mathrm{mol} \mathrm{CO}_{2} / \mu\right.$ mol ligand $)$. This small $\mathrm{CO}_{2}$ evolution from MIP$208 @ \mathrm{RuO}_{\mathrm{x}}$ compares favourably with the stability of the most robust MOFs reported as photocatalysts under similar irradiation conditions. ${ }^{82}$

The crystallinity of the MIP-208@ $\mathrm{RuO}_{\mathrm{x}}$ sample after seven catalytic cycles was wellmaintained, as no notable change in the PXRD pattern was observed (Figure S18). Furthermore, the solid-state ${ }^{13} \mathrm{C}-\mathrm{NMR}$ spectra of the fresh and seven-times reused MIP$208 @ \mathrm{RuO}_{\mathrm{x}}$ were coincident (Figure S19). DF-TEM images of the catalyst after recycling were also similar to those of the fresh sample, without any evidence of $\mathrm{RuO}_{\mathrm{x}}$ particle growth or agglomeration (Figure S20). Thus, both photocatalytic activity and characterization of the seven-times used MIP-208@ $\mathrm{RuO}_{\mathrm{x}}$ sample indicate the stability of the material under irradiation conditions.

To determine the origin of $\mathrm{CH}_{4}$, an experiment using ${ }^{13} \mathrm{C}$-labelled $\mathrm{CO}_{2}$ was carried out, monitoring the isotopic composition of $\mathrm{CH}_{4}$ evolved by mass spectrometry. The results presented in Figure S21 show the peaks corresponding to ${ }^{13} \mathrm{CH}_{4}$ appearing at 17 Dalton. 
It is important to note that injection of a sample before irradiation does not show in the chromatogram any peak at the retention time of ${ }^{13} \mathrm{CH}_{4}$.

To shed light about the main operating mechanism of the photocatalytic methanation, $\mathrm{H}_{2}$ was replaced by dimethylaniline as the sacrificial electron donor. Two possible operating mechanisms have been reported for the photocatalytic methanation of $\mathrm{CO}_{2}$, either the so-called photothermal pathway or the photoinduced charge separation pathway ${ }^{78}$. In the photothermal mechanism, light energy is converted into local heat on the metal NPs and the temperature increases on the NP surface promotes the thermal conversion of $\mathrm{CO}_{2}$ and $\mathrm{H}_{2}$ into the product. ${ }^{78}$ This local temperature increase is undetectable by measurements of the macroscopic system due to the low proportion of $\mathrm{RuO}_{\mathrm{x}}$. In the photocatalytic mechanism, light absorption leads to a charge separation with the generation of electrons in the conduction band and holes in the valence band that causes $\mathrm{CO}_{2}$ reduction and $\mathrm{H}_{2}$ oxidation, respectively. In the photoinduced charge separation state, the use of an electron donor better than $\mathrm{H}_{2}$ should equally result in $\mathrm{CO}_{2}$ conversion to methane, with an even higher reaction rate than using $\mathrm{H}_{2}$ as reagent. On the contrary, this electron donor should not be adequate for the photothermal reaction. In the present study, dimethylaniline $(0.76 \mathrm{~V}$ vs $\mathrm{Ag} / \mathrm{AgCl})$ was selected as electron donor. ${ }^{83}$ The results show that $\mathrm{CH}_{4}$ is formed in the presence of dimethylaniline $(40 \mu \mathrm{L})$ even at a higher rate than in the presence of $\mathrm{H}_{2}$ as a reagent, reaching a $\mathrm{CH}_{4}$ production rate in $5 \mathrm{~h}$ of $250 \mu \mathrm{mol} \mathrm{g}$ catalyst $^{-1}$ compared to $180 \mu \mathrm{mol} \mathrm{g}_{\text {catalyst }}{ }^{-1}$ when using $\mathrm{H}_{2}$ as a reagent. Interestingly, a similar experiment using anisole as electron donor with higher oxidation potential $(1.93 \mathrm{~V}$ vs $\mathrm{Ag} / \mathrm{AgCl})$ than dimethylaniline $(0.90 \mathrm{~V}$ vs $\mathrm{Ag} / \mathrm{AgCl})$ resulted in a lower $\mathrm{CH}_{4}$ production $\left(25 \mu \mathrm{mol} \mathrm{g}_{\text {catalyst }}{ }^{-1}\right)$. This observation is in agreement with previous report using $\mathrm{Cu}_{2} \mathrm{O}$ supported on graphene as photocatalyst and observing that the methane production rates decreases as the oxidation potential of the sacrificial electron donor increases ${ }^{84 ; 85}$. Furthermore, if photomethanation is attempted under the same conditions, but in the presence of nitrobenzene $(40 \mu \mathrm{L},-0.36 \mathrm{~V} \text { vs. } \mathrm{Ag} / \mathrm{AgCl})^{86}$ as electron quencher competing with $\mathrm{CO}_{2}$, then, no formation of $\mathrm{CH}_{4}$ is observed. This dependence of $\mathrm{CH}_{4}$ formation on the presence and redox potential of electron donor 
and acceptor quenchers is fully consistent with the prevalent operation of a photocatalytic mechanism involving photoinduced $\mathrm{e}^{-} / \mathrm{h}^{+}$separation and $\mathrm{CH}_{4}$ formation by consecutive eight $\mathrm{e}^{-}$(plus $8 \mathrm{H}^{+}$) reduction of $\mathrm{CO}_{2}$.

The occurrence of the photoinduced charge separation and the reaction of this photoinduced charge separated state with electron donors was additionally confirmed by photocurrent measurements. A thin film of MIP-208@ $\mathrm{RuO}$ was further deposited on a transparent FTO electrode. Starting from a polarization potential of $1.6 \mathrm{~V}$, the MIP$208 @ \mathrm{RuO}_{\mathrm{x}}$ electrode was submitted to a decreasing bias potential in a single electrochemical cell using tetrapropylammonium tetrafluoroborate in acetonitrile as electrolyte. The current density clearly increased at each voltage upon illumination of the photoanode with the output of a Xe lamp (Figure S22). Furthermore, the presence of methanol as an electron donor increases significantly the photocurrent, indicating that this reagent is acting as a sacrificial electron donor, becoming oxidized and donating electrons to the MIP-208@ $\mathrm{RuO}_{\mathrm{x}}$ in its excited state.

Besides the photocatalytic mechanism of $\mathrm{CO}_{2}$ reduction to $\mathrm{CH}_{4}$, there are reports in the literature showing that $\mathrm{CO}_{2}$ reduction can take place alternatively through a photothermal pathway ${ }^{87 ; 88}$. In this mechanism, photon energy is dissipated in the active site that undergoes a local heating at nanometer scale sufficient to overcome the activation barrier for a thermocatalytic pathway. The local temperature of these nanometric hot spots cannot be determined macroscopically, particularly considering the low proportion of these hot spots in the composition of the photocatalyst and the thermal conductivity of the medium. One common methodology to assess the occurrence of local heating is the use of quantum dots (QDs) monitoring the emission intensity decrease as local thermometer ${ }^{84}$. This method is based on the decrease of the emission intensity of quantum dots as the temperature experienced by nanoparticle increases in a certain temperature range. In a preliminary calibration study, it was determined that the photoluminescence lifetime emission of CdSe-ZnS QDs decreases as the temperature increases (Figure S23). This observation is a consequence of the emission intensity decrease of core-shell CdSe-ZnS nanoparticles as the temperature 
increases in the range from 25 to $200{ }^{\circ} \mathrm{C}$ (Figure S24). Thus, core-shell CdSe-ZnS nanoparticles can be used as local, nanometric thermometer to get some information about local temperature changes during irradiation that could distinguish between photocatalytic or photothermal reaction mechanism when using MIP-208@RuOx in photomethanation. After calibration, the $\mathrm{CdSe}-\mathrm{ZnS}$ nanoparticles were dispersed on the MIP-208@ $\mathrm{RuO}_{\mathrm{x}}$ sample. Comparison of the emission intensity of CdSe-ZnS adsorbed on MIP-208@RuOx depending on whether the solid is in the dark or illuminated with the Xe lamp conclusively shows that, even if the macroscopic temperature is supposed to be constant, CdSe-ZnS QDs experience a local heating under Xe irradiation, required for a photothermal mechanism (Figure S25). However, it has to be commented that although the experiments with $\mathrm{CdSe}-\mathrm{ZnS}$ as local temperature probes conclusively show the temperature increase at the nanometric scale and not observed macroscopically, the previously commented quenching experiments and particularly the fact that a good electron acceptor completely stops methane formation indicates that the mechanism is fully photocatalytic derived from charge-separation, but accompanied with temperature increase at the nanoparticles that does not cause product formation. Otherwise, nitrobenzene, quenching electrons but not impeding heating, would have not completely stop $\mathrm{CH}_{4}$ formation.

The influence of the temperature was addressed by performing photomethanation by MIP-208@ $\mathrm{RuO}_{\mathrm{x}}$ at different temperatures in the range from 100 to $200{ }^{\circ} \mathrm{C}$ (Figure S26). It was observed that $\mathrm{CH}_{4}$ formation starts at $100{ }^{\circ} \mathrm{C}$, but undergoes a significant formation rate increase at temperatures higher than $150{ }^{\circ} \mathrm{C}$. Control experiments in the dark indicate that $\mathrm{CH}_{4}$ formation is one order of magnitude less or lower than upon irradiation. This behavior indicates that the photocatalytic process has an activation energy.

To understand the origin of the thermal activation of the photocatalytic reaction, the possible role of $\mathrm{H}_{2} \mathrm{O}$ as a poison was considered. $\mathrm{H}_{2} \mathrm{O}$ is a reaction product that can be strongly adsorbed on the active sites competing favorably with $\mathrm{CO}_{2}$ adsorption and stopping the reaction. In this case, one of the roles of heating would be $\mathrm{H}_{2} \mathrm{O}$ desorption 
from the active sites. Aimed at providing some support to this proposal, a control experiment was performed adding $\mathrm{H}_{2} \mathrm{O}(60 \mu \mathrm{L})$ at the initial reaction stage of the photocatalytic methanation. A significant decrease in the initial methanation rate by one order of magnitude from 40 to about $4 \mu \mathrm{mol} \mathrm{g}_{\text {catalyst }}{ }^{-1} \mathrm{~h}^{-1}$ was measured (Figure S26). This negative role of $\mathrm{H}_{2} \mathrm{O}$ should also take place in the reactor as photomethanation starts and temperatures above $100{ }^{\circ} \mathrm{C}$ would allow its desorption.

In summary, benefiting from a synergetic adjustment including both the preformed $\mathrm{Ti}_{8} \mathrm{AF}$ cluster rearrangement and the in situ linker formation, which slows down the reaction, MIP-208, the first Ti-IPA MOF, was synthesized with high crystallinity and tunable scale. Its crystal structure, which is isostructural to CAU-10, constitutes cisconnected corner-sharing $\mathrm{TiO}_{6}$ polyhedra that extend along the $c$-axis resulting in a $1 \mathrm{D}$ helical chain inorganic building unit. Multivariate MIP-208 structures with tunable chemical environments and sizable porosities could be achieved by adopting the solidsolution mixed-linker synthesis strategy. This material has been proven to be the best photocatalyst for the methanation of $\mathrm{CO}_{2}$ in terms of activity and selectivity among all the pure MOFs, owing to its excellent stability and photoresponse. Further improvement of catalytic activity was achieved by the photodeposition of $\mathrm{RuO}_{\mathrm{x}}$. The resulting MIP-208@ $\mathrm{RuO}_{\mathrm{x}}$ composite exhibits, under identical conditions, an enhancement of efficiency of a factor of about 20 in comparison with that of the pure MOF. The composite catalyst displays a high stability and reusability. These results illustrate the continuous interest in the synthesis of novel Ti-MOFs that could lead to improved generations of photocatalysts, suitable for the production of solar fuels and the photoinduced methanation of $\mathrm{CO}_{2}$. 


\section{EXPERIMENTAL PROCEDURES}

\section{Resource Availability}

\section{Lead Contact}

Further information and requests for resources could be directed to and will be fulfilled by the Lead Contact, Sujing Wang (sjwang4@ustc.edu.cn).

\section{Materials Availability}

All chemicals were purchased from commercial suppliers and used as received without further purification. 5- $\mathrm{NH}_{2}$-IPA (Alfa Aesar), IPA (Alfa Aesar), 5-Me-IPA (TCI), 5-Br-IPA (Sigma), 5F-IPA (FluoroChem), 5-tBu-IPA (FluoroChem), PDA (FluoroChem), formic acid (Fisher), acetic acid (Acros), acetic anhydride (Sigma), $\mathrm{MeOH}$ (Fisher), Ti(iPrO) 4 (Alfa Aesar).

\section{Data and Code Availability}

Data supporting the findings of this paper are available from the corresponding authors upon reasonable request.

\section{Method Details}

Synthesis of MIP-208 (small scale)

To a $23 \mathrm{~mL}$ Teflon reactor, $\mathrm{Ti}_{8} \mathrm{AF}$ cluster solid $(220 \mathrm{mg})$ was added followed by adding acetic anhydride $(5 \mathrm{~mL})$ and acetic acid $(5 \mathrm{~mL})$. The mixture was stirred at room temperature for 20 minutes before 5- $\mathrm{NH}_{2}$-IPA (362 mg, $2 \mathrm{mmol}$ ) was added once. After stirring for 10 minutes, $\mathrm{MeOH}(0.5 \mathrm{~mL})$ was added and the overall mixture was stirred at room temperature for another 10 minutes. Afterwards, the reaction was heated in an oven at $180{ }^{\circ} \mathrm{C}$ for 48 hours. When the reaction was cooled to room temperature, the dark yellow/light brown product was collected by filtration, washed with acetone then air dried. Yield: $330 \mathrm{mg}$ (average of five parallel reactions).

Synthesis of MIP-208 (scale-up)

To a $125 \mathrm{~mL}$ Teflon reactor, $\mathrm{Ti}_{8} \mathrm{AF}$ cluster solid ( $\left.3.5 \mathrm{~g}\right)$ was added followed by adding acetic 
anhydride $(25 \mathrm{~mL})$ and acetic acid $(25 \mathrm{~mL})$. The mixture was stirred at room temperature for 20 minutes before 5- $\mathrm{NH}_{2}$-IPA (3.62 g, $\left.20 \mathrm{mmol}\right)$ was added once. After stirring for 10 minutes, $\mathrm{MeOH}(3.5 \mathrm{~mL})$ was added and the overall mixture was stirred at room temperature for another 10 minutes. Afterwards, the reaction was heated in an oven at $180{ }^{\circ} \mathrm{C}$ for 48 hours. When the reaction was cooled to room temperature, the dark yellow/light brown product was collected by filtration, washed with acetone than air dried. Yield: $4.3 \mathrm{~g}$ (average of five parallel reactions).

\section{Typical synthesis of MTV-MIP-208}

To a $23 \mathrm{~mL}$ Teflon reactor, $\mathrm{Ti}_{8} \mathrm{AF}$ cluster solid $(220 \mathrm{mg})$ was added followed by adding acetic anhydride $(5 \mathrm{~mL})$ and acetic acid $(5 \mathrm{~mL})$. The mixture was stirred at room temperature for 20 minutes before a linker mixture of 5- $\mathrm{NH}_{2}$-IPA $(1 \mathrm{mmol})$ and the secondary IPA ligand $(1 \mathrm{mmol})$ was added. After stirring for 10 minutes, $\mathrm{MeOH}(0.5 \mathrm{~mL})$ was added and the overall mixture was stirred at room temperature for another 10 minutes. Afterwards, the reaction was heated in an oven at $180{ }^{\circ} \mathrm{C}$ for 48 hours. When the reaction was cooled to room temperature, the dark yellow/light brown product was collected by filtration, washed with acetone then air dried.

\section{Photocatalytic methanation tests}

The photocatalytic methanation of $\mathrm{CO}_{2}$ experiments were carried out using a quartz photoreactor $(51 \mathrm{~mL})$ equipped with a heating mantle to control the desired temperature. In a typical experiment the powdered photocatalyst $(15 \mathrm{mg})$ is placed as a bed in the reactor, and then the system purged first with $\mathrm{H}_{2}$ and later with $\mathrm{CO}_{2}$ until a ratio of 4 to 1 is obtained. Subsequently, the photoreactor is heated at $200{ }^{\circ} \mathrm{C}$, and then the photocatalyst is irradiated using a Xe lamp $(150 \mathrm{~W})$ or a solar simulator. At the desired reaction time, an aliquot was sampled from the photoreactor and analyzed in an Agilent 490 MicroGC equipped with two channels and thermal conductivity detectors. One channel equipped with a MolSieve $5 \AA$ column allows analysis of $\mathrm{H}_{2}, \mathrm{O}_{2}, \mathrm{~N}_{2}$ and $\mathrm{CO}$, while the other channel equipped with a Pore Plot Q column allows determining $\mathrm{CO}_{2}, \mathrm{CH}_{4}$ and short chain hydrocarbons. Quantification was performed using calibration plots employing commercially available gas mixtures.

\section{X-ray crystallographic data}

The single crystal X-ray crystallographic data for MIP-208 were collected on the PROXIMA 
2A beamline at Synchrotron SOLEIL and have been deposited at the Cambridge Crystallographic Data Centre (CCDC), under deposition number CCDC 1989406. Highresolution PXRD data of MIP-208 for Rietveld refinement were collected on the STOE STADI PESSENTIAL X-ray diffractometer equipped with a Mythen II detector in the Debye-Scherrer mode with pure $\mathrm{Cu} \mathrm{K} \alpha 1$ radiation $(\lambda=1.5406 \mathrm{~A})$ (capillary: $0.2 \mathrm{~mm}$, angle range: $5-120^{\circ}$, step size: $0.015^{\circ}$, total counting time: $16.5 \mathrm{~h}$, room temperature), and have been deposited at CCDC under the deposition number CCDC 2011709. These data can be obtained free of charge from the CCDC database via www.ccdc.cam.ac.uk. All other relevant data supporting the findings of this study are available from the corresponding authors on request.

\section{ACKNOWLEDGEMENTS}

S.W. acknowledges the support from the National Natural Science Foundation of China (22071234) and the Fundamental Research Funds for the Central Universities. S.N. thanks financial support by Ministerio de Ciencia, Innovación y Universidades RTI2018-099482-AI00 project, the Fundación Ramón Areces (XVIII Concurso Nacional para la Adjudicación de Ayudas a la Investigación en Ciencias de la Vida y de la Materia, 2016) and Generalitat Valenciana grupos de investigación consolidables 2019 (AICO/2019/214) project. C-C.C. acknowledges the support from the Program of China Scholarship Council (201700260093) and PHC Cai YuanPei Project (38893VJ). C.M-C is grateful for financial support from the Institut Universitaire de France (IUF) and the Paris Ile-de- France Region - DIM "Respore". H.G. thanks financial support to the Spanish Ministry of Science and Innovation (Severo Ochoa and RTI2018-098237-CO2-1) and Generalitat Valenciana (Prometeo2017/083). The authors thank the staff at Synchrotron SOLEIL and the associated scientists for beamtime and assistance during SCXRD data collections on PROXIMA 2A, as well as Dr. Peng Guo and Dr. Nana Yan from Dalian Institute of Chemical Physics (Chinese Academy of Sciences) for the collection of high-resolution PXRD data for Rietveld refinement.

\section{AUTHOR CONTRIBUTIONS}


Conceptualization, S.W., C.S. S.N. and H.G.; Investigation, S.W., M.C-A., S.N., C-C.C., A.T., J.M., C.M.-C., L.Y., H.W., W.S., H.G., and C.S.; Writing - Original Draft, S.W.; Writing Review \& Editing, S.W., S.N., A.T., C.M.-C., W.S. H.G., and C.S. Supervision, H.G., and C.S.

\section{DECLARATION OF INTERESTS}

The authors declare no competing interests.

\section{Main Figure titles and legend}

Figure 1. Comparison of 1D Ti-O inorganic building units reported.

(A) Linear TiO chain in MIL-91.

(B) Linear $\mathrm{Ti}_{6} \mathrm{O}_{9}$ nanowire in MIP-177-HT, ZSTU-1, 2 and 3.

(C) Linear TiO chain in DGIST-1 and ACM-1.

(D) Helical TiO chain in MIP-208 reported here.

Figure 2. Crystal structure of MIP-208.

(A) Coordination environment of the $\mathrm{Ti}^{4+}$ ions and arrangement of 5-Aa-IPA linkers around them.

(B) Structure viewed along the $b$-axis.

(C) Structure viewed along the $c$-axis, showing $1 \mathrm{D}$ channels ( $\mathrm{Ti}$ in purple, $\mathrm{C}$ in gray, $\mathrm{O}$ in red, and the blue ball represents the acetamide group, all hydrogen atoms are omitted for clarity).

Figure 3. Illustration and characterizations of MTV-MIP-208 materials.

(A) Illustration of MTV-MIP-208 structure with multiple functional groups (the blue ball represents the acetamide group, the green ball represents the other functional groups. Hydrogen atoms are omitted for clarity).

(B) PXRD patterns of the parent and MTV-MIP-208s $\left(\lambda_{\mathrm{Cu}} \approx 1.5406 \AA\right)$.

(C) $\mathrm{CO}_{2}$ adsorption isotherms collected at $273 \mathrm{~K}$ for parent and MTV-MIP-208 solids.

Figure 4. Results of water stability tests.

(A) PXRD patterns for the MIP-208 samples before and after water treatments.

(B) $\mathrm{CO}_{2}$ adsorption isotherms collected at $298 \mathrm{~K}$ for the MIP-208 samples before and after water 
treatments.

Figure 5. Photocatalytic performance of MIP-208@ $\mathrm{RuO}_{\mathrm{x}}$ in $\mathrm{CO}_{2}$ methanation.

(A) Temporal evolution of $\mathrm{CH}_{4}$ during the photocatalytic $\mathrm{CO}_{2}$ reduction with MIP-208@ $\mathrm{RuO}$ as the catalyst using a solar simulator, a xenon lamp with full range lights and visible light (Xe lamp with $>455$ nm filter).

(B) Reusability of MIP-208@ $\mathrm{RuO}_{\mathrm{x}}$ in the photocatalytic methanation of $\mathrm{CO}_{2}$ (seven-times cycling) using a xenon lamp as irradiation source. Reaction conditions: catalyst (15 mg), $\mathrm{H}_{2}(1.05 \mathrm{bar}), \mathrm{CO}_{2}(0.25 \mathrm{bar})$, temperature $\left(200^{\circ} \mathrm{C}\right)$, irradiation source a xenon lamp $\left(1350 \mathrm{~W} \times \mathrm{m}^{-2}\right)$ or solar simulator $\left(1000 \mathrm{~W} \times \mathrm{m}^{-2}\right)$.

\section{REFERENCES}

1. Dhakshinamoorthy A., Li Z., and Garcia H. (2018). Catalysis and photocatalysis by metal organic frameworks. Chem Soc Rev 47, 8134-8172.

2. Chen L., and Xu Q. (2019). Metal-Organic Framework Composites for Catalysis. Matter 1, 57-89.

3. García H., and Navalón S. (2018). In Metal-Organic Frameworks: Applications in Separations and Catalysis (Wiley - VCH Verlag GmbH \& Co. KGaA).

4. Yeung H. H., Li W., Saines P. J., Koster T. K., Grey C. P., and Cheetham A. K. (2013). Ligand-directed control over crystal structures of inorganic-organic frameworks and formation of solid solutions. Angew Chem Int Ed 52, 5544-5547.

5. Lu W., Wei Z., Gu Z. Y., Liu T. F., Park J., Park J., et al. (2014). Tuning the structure and function of metal-organic frameworks via linker design. Chem Soc Rev 43, 5561-5593.

6. Desai A. V., Sharma S., Let S., and Ghosh S. K. (2019). N-donor linker based metal-organic frameworks (MOFs): Advancement and prospects as functional materials. Coord Chem Rev 395, 146-192.

7. Zhang H., Zou R., and Zhao Y. (2015). Macrocycle-based metal-organic frameworks. Coord Chem Rev 292, 74-90.

8. He Y., Li B., 0’Keeffe M., and Chen B. (2014). Multifunctional metal-organic frameworks constructed from meta-benzenedicarboxylate units. Chem Soc Rev 43, 56185656.

9. Wang H., Zhu Q.-L., Zou R., and Xu Q. (2017). Metal-Organic Frameworks for Energy Applications. Chem 2, 52-80.

10. Kuppler R. J., Timmons D. J., Fang Q. -R., Li J.-R., Makal T. A., Young M. D., et al. (2009). Potential applications of metal-organic frameworks. Coord Chem Rev 253, 3042-3066.

11. Czaja A. U., Trukhan N., and Muller U. (2009). Industrial applications of metalorganic frameworks. Chem Soc Rev 38, 1284-1293.

12. Silva P., Vilela S. M., Tome J. P., and Almeida Paz F. A. (2015). Multifunctional 
metal-organic frameworks: from academia to industrial applications. Chem Soc Rev 44, 6774-6803.

13. Ren J., Dyosiba X., Musyoka N. M., Langmi H. W., Mathe M., and Liao S. (2017). Review on the current practices and efforts towards pilot-scale production of metalorganic frameworks (MOFs). Coord Chem Rev 352, 187-219.

14. Hillesheim P. M. L. F. K. T. W. E. R. J. N. B. W. (2007). Phthalic Acid and Derivatives. In Ullmann's Encyclopedia of Industrial Chemistry.

15. Sheehan R. J. (2011). Terephthalic Acid, Dimethyl Terephthalate. In U11mann's Encyclopedia of Industrial Chemistry.

16. Ohtani M., Takase K., Wang P., Higashi K., Ueno K., Yasuda N., et al. (2016). Water-triggered macroscopic structural transformation of a metal - organic framework. CrystEngComm 18, 1866-1870.

17. Reinsch H., De Vos D., and Stock N. (2013). Structure and Properties of [A14(OH) 8 (o-C6H4 (CO2)2)2] • H20, a Layered Aluminum Phthalate. Z Anorg Allg Chem 639, 2785-2789.

18. Li H., Davis C. E., Groy T. L., Kelley D. G., and Yaghi 0. M. (1998). Coordinatively Unsaturated Metal Centers in the Extended Porous Framework of Zn3 (BDC) 3 - 6CH3OH (BDC = 1, 4-Benzenedicarboxylate). J Am Chem Soc 120, 2186-2187.

19. Banerjee D., and Parise J. B. (2011). Recent Advances in s-Block Metal Carboxylate Networks. Cryst Growth Des 11, 4704-4720.

20. Pagis C., Ferbinteanu M., Rothenberg G., and Tanase S. (2016). Lanthanide-Based Metal Organic Frameworks: Synthetic Strategies and Catalytic Applications. ACS Catal 6 , 6063-6072.

21. Aguirre-Díaz L. M., Reinares-Fisac D., Iglesias M., Gutiérrez-Puebla E., Gándara F., Snejko N., et al. (2017). Group 13th metal-organic frameworks and their role in heterogeneous catalysis. Coord Chem Rev 335, 1-27.

22. Kang M. P., Luo D. B., Deng Y. R., Li R. X., and Lin Z. E. (2014). Solvothermal synthesis and characterization of new calcium carboxylates based on cluster- and rod-1ike building blocks. Inorg Chem Commun 47, 52-55.

23. Bourne S. A., Lu J. J., Mondal A., Moulton B., and Zaworotko M. J. (2001). Selfassembly of nanometer-scale secondary building units into an undulating twodimensional network with two types of hydrophobic cavity. Angew Chem Int Ed 40, 21112113.

24. Vodak D. T., Braun M. E., Kim J., Eddaoudi M., and Yaghi 0. M. (2001). Metalorganic frameworks constructed from pentagonal antiprismatic and cuboctahedral secondary building units. Chem Commun, 2534-2535.

25. Barthelet K., Riou D., and Ferey G. (2002). [V(III) (H20)]30(02CC6H4CO2) 3. (Cl, 9H20) (MIL-59): a rare example of vanadocarboxylate with a magnetically frustrated three-dimensional hybrid framework. Chem Commun, 1492-1493.

26. Qazvini 0. T., Babarao R., Shi Z. L., Zhang Y. B., and Telfer S. G. (2019). A Robust Ethane-Trapping Metal-Organic Framework with a High Capacity for Ethylene Purification. J Am Chem Soc 141, 5014-5020.

27. Kim J. -Y., Norquist A. J., and 0' Hare D. (2003). Incorporation of uranium(vi) into metal - organic framework solids, [U02(C4H404)] • H20, [U02F (C5H604)] • 2H20, and 
[(UO2) 1. 5 (C8H404) 2]2 [ (CH3) 2NCOH2] • H20. Dalton Trans, 2813-2814.

28. Wang G., Song T., Fan Y., Xu J., Wang M., Zhang H., et al. (2010). [Y2 (H20) (BDC) 3 (DMF) ] (DMF) 3: A rare 2-D (42.6) (45.6)2(48.62) (49.65.8) net with multi-helical-array and opened windows. Inorg Chem Commun 13, 502-505.

29. Mihalcea I., Henry N., Clavier N., Dacheux N., and Loiseau T. (2011). Occurence of an octanuclear motif of uranyl isophthalate with cation-cation interactions through edge-sharing connection mode. Inorg Chem 50, 6243-6249.

30. Vougo-Zanda M., Wang X., and Jacobson A. J. (2007). Influence of ligand geometry on the formation of In-0 chains in metal-oxide organic frameworks (MOOFs). Inorg Chem 46, 8819-8824.

31. Bu F., and Xiao S. J. (2010). A 4-connected anionic metal-organic nanotube constructed from indium isophthalate. CrystEngComm 12, 3385-3387.

32. Panda T., Kundu T., and Banerjee R. (2013). Structural isomerism leading to variable proton conductivity in indium(III) isophthalic acid based frameworks. Chem Commun 49, 6197-6199.

33. Chen P. K., Che Y. X., Zheng J. M., and Batten S. R. (2007). Heteropolynuclear metamagnet showing spin canting and single-crystal to single-crystal phase transformation. Chem Mat 19, 2162-2167.

34. Zhang L., Qin Y. Y., Li Z. J., Lin Q. P., Cheng J. K., Zhang J., et al. (2008). Topology analysis and nonlinear-optical-active properties of luminescent metalorganic framework materials based on zinc/lead isophthalates. Inorg Chem 47, 82868293.

35. Zhang J. P., Ghosh S. K., Lin J. B., and Kitagawa S. (2009). New heterometallic carboxylate frameworks: synthesis, structure, robustness, flexibility, and porosity. Inorg Chem 48, 7970-7976.

36. McCormick L. J., Morris S. A., Slawin A. M. Z., Teat S. J., and Morris R. E. (2016). Coordination Polymers of 5-Alkoxy Isophthalic Acids. Cryst Growth Des 16, 5771-5780.

37. Chen J., Li C. -P., and Du M. (2011). Substituent effect of R-isophthalates ( $\mathrm{R}=$ $-\mathrm{H},-\mathrm{CH} 3,-\mathrm{OCH} 3,-\mathrm{tBu},-\mathrm{OH}$, and - NO2) on the construction of CdII coordination polymers incorporating a dipyridyl tecton 2,5-bis(3-pyridyl)-1,3,4-oxadiazole. CrystEngComm 13, 1885-1893.

38. Du M., Zhang Z.-H., You Y.-P., and Zhao X. -J. (2008). R-Isophthalate (R $=-H$, - N02, and - $\mathrm{COOH}$ ) as modular building blocks for mixed-ligand coordination polymers incorporated with a versatile connector 4-amino-3, 5-bis(3-pyridy1)-1,2, 4-triazole. CrystEngComm 10, 306-321.

39. Chen L., Ye J.-W., Wang H. -P., Pan M., Yin S. -Y., Wei Z.-W., et al. (2017). Ultrafast water sensing and thermal imaging by a metal-organic framework with switchable luminescence. Nat Commun 8, 15985.

40. Yuan S., Qin J.-S., Lollar C. T., and Zhou H. -C. (2018). Stable Metal-Organic Frameworks with Group 4 Metals: Current Status and Trends. ACS Central Sci 4, 440450.

41. Rieth A. J., Wright A. M., and Dinca M. (2019). Kinetic stability of metalorganic frameworks for corrosive and coordinating gas capture. Nat Rev Mater 4, 708- 
725.

42. Dhakshinamoorthy A., Asiri A. M., and Garcia H. (2016). Metal-Organic Framework (MOF) Compounds: Photocatalysts for Redox Reactions and Solar Fuel Production. Angew Chem Int Ed 55, 5414-5445.

43. Alvaro M., Carbonell E., Ferrer B., Llabres i Xamena F. X., and Garcia H. (2007). Semiconductor behavior of a metal-organic framework (MOF). Chem Eur J 13, 5106-5112. 44. Nasalevich M. A., Goesten M. G., Savenije T. J., Kapteijn F., and Gascon J. (2013). Enhancing optical absorption of metal-organic frameworks for improved visible light photocatalysis. Chem Commun 49, 10575-10577.

45. Zhu J. J., Li P. Z., Guo W. H., Zhao Y. L., and Zou R. Q. (2018). Titanium-based metal-organic frameworks for photocatalytic applications. Coord Chem Rev 359, 80101.

46. Benoit V., Pillai R. S., Orsi A., Normand P., Jobic H., Nouar F., et al. (2016). MIL-91(Ti), a small pore metal - organic framework which fulfils several criteria: an upscaled green synthesis, excellent water stability, high C02 selectivity and fast C02 transport. J Mater Chem A 4, 1383-1389.

47. Sun Y., Liu Y., Caro J., Guo X., Song C., and Liu Y. (2018). In-Plane Epitaxial Growth of Highly c-0riented NH2-MIL-125(Ti) Membranes with Superior H2/C02 Selectivity. Angew Chem Int Ed 57, 16088-16093.

48. Wahiduzzaman M., Wang S. J., Schnee J., Vimont A., Ortiz V., Yot P. G., et al. (2019). A High Proton Conductive Hydrogen-Sulfate Decorated Titanium Carboxylate Metal-Organic Framework. Acs Sustain Chem Eng 7, 5776-5783.

49. Pinto R. V., Wang S., Tavares S. R., Pires J., Antunes F., Vimont A., et al. (2020). Tuning Cellular Biological Functions Through the Controlled Release of N0 from a Porous Ti-MOF. Angew Chem Int Ed 59, 5135-5143.

50. Assi H., Mouchaham G., Steunou N., Devic T., and Serre C. (2017). Titanium coordination compounds: from discrete metal complexes to metal-organic frameworks. Chem Soc Rev 46, 3431-3452.

51. Tachikawa T., Tojo S., Fujitsuka M., Sekino T., and Majima T. (2006). Photoinduced charge separation in titania nanotubes. J Phys Chem B 110, 14055-14059. 52. Wang S., Kitao T., Guillou N., Wahiduzzaman M., Martineau-Corcos C., Nouar F., et al. (2018). A phase transformable ultrastable titanium-carboxylate framework for photoconduction. Nat Commun 9, 1660.

53. Serre C., Groves J. A., Lightfoot P., Slawin A. M. Z., Wright P. A., Stock N., et al. (2006). Synthesis, Structure and Properties of Related MicroporousN, N 'Piperazinebismethylenephosphonates of Aluminum and Titanium. Chem Mat 18, 1451-1457. 54. Li C., Xu H., Gao J., Du W., Shangguan L., Zhang X., et al. (2019). Tunable titanium metal - organic frameworks with infinite 1D Ti - 0 rods for efficient visiblelight-driven photocatalytic H2 evolution. J Mater Chem A 7, 11928-11933.

55. Keum Y., Park S., Chen Y.-P., and Park J. (2018). Titanium-Carboxylate Meta1Organic Framework Based on an Unprecedented Ti-0xo Chain Cluster. Angew Chem Int Ed 57, $14852-14856$.

56. Yuan S., Liu T.-F., Feng D., Tian J., Wang K., Qin J., et al. (2015). A single crystalline porphyrinic titanium metal-organic framework. Chem Sci 6, 3926-3930. 
57. Padial N. M., Castells-Gil J., Almora-Barrios N., Romero-Angel M., da Silva I., Barawi M., et al. (2019). Hydroxamate Titanium-Organic Frameworks and the Effect of Siderophore-Type Linkers over Their Photocatalytic Activity. J Am Chem Soc 141, 13124-13133.

58. Wang S., Reinsch H., Heymans N., Wahiduzzaman M., Martineau-Corcos C., De Weireld G., et al. (2020). Toward a Rational Design of Titanium Metal-Organic Frameworks. Matter 2, 440-450.

59. Hendon C. H., Tiana D., Fontecave M., Sanchez C., D’Arras L., Sassoye C., et al. (2013). Engineering the optical response of the titanium-MIL-125 metal-organic framework through ligand functionalization. J Am Chem Soc 135, 10942-10945.

60. Fu Y., Sun D., Chen Y., Huang R., Ding Z., Fu X., et al. (2012). An AmineFunctionalized Titanium Metal - Organic Framework Photocatalyst with Visible-LightInduced Activity for C02 Reduction. Angew Chem Int Ed 51, 3364-3367.

61. Duran D., Couster S. L., Desjardins K., Delmotte A., Fox G., Meijers R., et al. (2013). PROXIMA 2A - A New Fully Tunable Micro-focus Beamline for Macromolecular Crystallography. J Phys Conf Ser 425, 012005.

62. Reinsch H., van der Veen M. A., Gil B., Marszalek B., Verbiest T., de Vos D., et al. (2013). Structures, Sorption Characteristics, and Nonlinear Optical Properties of a New Series of Highly Stable Aluminum MOFs. Chem Mat 25, 17-26.

63. Ferey G., and Serre C. (2009). Large breathing effects in three-dimensional porous hybrid matter: facts, analyses, rules and consequences. Chem Soc Rev 38, 13801399.

64. Férey G. (2016). Giant flexibility of crystallized organic - inorganic porous solids: facts, reasons, effects and applications. New J Chem 40, 3950-3967.

65. Leshuk T., Parviz R., Everett P., Krishnakumar H., Varin R. A., and Gu F. (2013). Photocatalytic Activity of Hydrogenated Ti02. ACS App1 Mater Interfaces 5, 1892-1895. 66. Chen X., Liu L., and Huang F. (2015). Black titanium dioxide (Ti02) nanomaterials. Chem Soc Rev 44, 1861-1885.

67. Liu L., and Chen X. (2014). Titanium dioxide nanomaterials: self-structural modifications. Chem Rev 114, 9890-9918.

68. Reinsch H., Waitschat S., and Stock N. (2013). Mixed-1inker MOFs with CAU-10 structure: synthesis and gas sorption characteristics. Dalton Trans 42, 4840-4847.

69. Deng H., Doonan C. J., Furukawa H., Ferreira R. B., Towne J., Knobler C. B., et al. (2010). Multiple Functional Groups of Varying Ratios in Metal-Organic Frameworks. Science 327, 846-850.

70. Foo M. L., Matsuda R., and Kitagawa S. (2014). Functional Hybrid Porous Coordination Polymers. Chem Mat 26, 310-322.

71. Helal A., Yamani Z. H., Cordova K. E., and Yaghi 0. M. (2017). Multivariate metal-organic frameworks. Nat1 Sci Rev 4, 296-298.

72. Ding M., Flaig R. W., Jiang H. L., and Yaghi 0. M. (2019). Carbon capture and conversion using metal-organic frameworks and MOF-based materials. Chem Soc Rev 48, 2783-2828.

73. Li R., Hu J., Deng M., Wang H., Wang X., Hu Y., et al. (2014). Integration of an inorganic semiconductor with a metal-organic framework: a platform for enhanced 
gaseous photocatalytic reactions. Adv Mater 26, 4783-4788.

74. Cabrero-Antonino M., Remiro-Buenamanana S., Souto M., Garcia-Valdivia A. A., Choquesillo-Lazarte D., Navalon S., et al. (2019). Design of cost-efficient and photocatalytically active $\mathrm{Zn}$-based MOFs decorated with Cu20 nanoparticles for C02 methanation. Chem Commun 55, 10932-10935.

75. Ulmer U., Dingle T., Duchesne P. N., Morris R. H., Tavasoli A., Wood T., et al. (2019). Fundamentals and applications of photocatalytic C02 methanation. Nat Commun 10, 3169.

76. Younas M., Kong L. L., Bashir M. J. K., Nadeem H., Shehzad A., and Sethupathi S. (2016). Recent Advancements, Fundamental Challenges, and Opportunities in Catalytic Methanation of C02. Energ Fuel 30, 8815-8831.

77. Mateo D., Albero J., and García H. (2019). Titanium-Perovskite-Supported Ru02 Nanoparticles for Photocatalytic C02 Methanation. Joule 3, 1949-1962.

78. Wenderich K., and Mul G. (2016). Methods, Mechanism, and Applications of Photodeposition in Photocatalysis: A Review. Chem Rev 116, 14587-14619.

79. Giang T. P. L., Tran T. N. M., and Le X. T. (2012). Preparation and characterization of titanium dioxide nanotube array supported hydrated ruthenium oxide catalysts. Adv Nat Sci-Nanosci Nanotechnol 3, 015008.

80. Morgan D. J. (2015). Resolving ruthenium: XPS studies of common ruthenium materials. Surf Interface Anal 47, 1072-1079.

81. Albero J., Peng Y., and Garcia H. (2020). Photocatalytic C02 Reduction to C2+Products. Acs Catal 10, 5734-5749.

82. Mateo D., Santiago-Portillo A., Albero J., Navalon S., Alvaro M., and Garcia H. (2019). Long-Term Photostability in Terephthalate Metal-Organic Frameworks. Angew Chem Int Ed 58, 17843-17848.

83. Mateo D., Albero J., and García H. (2018). Graphene supported Ni0/Ni nanoparticles as efficient photocatalyst for gas phase C02 reduction with hydrogen. Appl Catal B: Environ 224, 563-571.

84. Mateo D., Albero J., and Garcia H. (2017). Photoassisted methanation using Cu20 nanoparticles supported on graphene as a photocatalyst. Energ Environ Sci 10, 23922400 .

85. Mateo D., Asiri A. M., Albero J., and Garcia H. (2018). The mechanism of photocatalytic C02 reduction by graphene-supported Cu20 probed by sacrificial electron donors. Photochem Photobiol Sci 17, 829-834.

86. Karthik R., Govindasamy M., Chen S. M., Mani V., Umamaheswari R., and Balamurugan T. S. T. (2016). Electrochemical Study of Nitrobenzene Reduction Using Potentiostatic Preparation of nephrolepis Leaf Like Silver Microstructure. Int J Electrochem Sc 11, 6164-6172.

87. Ulmer U., Dingle T., Duchesne P. N., Morris R. H., Tavasoli A., Wood T., et al. (2019). Fundamentals and applications of photocatalytic C02 methanation. Nat Commun 10, 3169.

88. Li X., Yu J., Jaroniec M., and Chen X. (2019). Cocatalysts for Selective Photoreduction of C02 into Solar Fuels. Chem Rev 119, 3962-4179. 
